\title{
Wavelets Cíclicas de Shannon e Aplicações em Processamento de Imagem
}

\author{
G. J. da Silva Jr. e R. M. Campello de Souza
}

\begin{abstract}
Resumo-Este trabalho introduz as wavelets cíclicas de Shannon. O esquema de codificação de imagem utilizando banco de filtros cíclicos é revisto utilizando-se essas wavelets. São apresentadas aplicações em interpolação cíclica e os resultados são comparados com a interpolação pela transformada discreta do cosseno tipo II. O conceito de filtro chave é introduzido utilizandose filtros cíclicos de Shannon, com potenciais aplicações em codificação de imagem, marca d'água e esteganografia.
\end{abstract}

Palavras-Chave-Wavelets cíclicas, banco de filtros cíclicos, esteganografia, multirresolução, wavelets cíclicas de Shannon.

Abstract-This paper introduces the Shannon cyclic wavelets and presents some of its applications in image processing. The image coding scheme based on cyclic filter banks is revisited using these wavelets. Applications in cyclic interpolation are presented and the results are compared with the interpolation based on the type II discrete cosine transform. The concept of key filter is introduced using Shannon cyclic filters, with potential applications in image coding, watermark and steganography.

Keywords - Cyclic wavelets, cyclic filter banks, steganography, multiresolution, Shannon cyclic wavelet.

\section{INTRODUÇÃO}

B ANCO de filtros e análise multirresolução via wavelets são ferramentas bem estabelecidas para o processamento digital de sinais com comprimento infinito [1]-[3]. Vaidyanathan e Kirac desenvolveram a teoria de banco de filtros cíclicos [4]-[6], a qual analisa sinais e sistemas com comprimento finito e fixo. Wavelets cíclicas podem também ser definidas utilizando-se essas estruturas [7], [8]. Nesse cenário, a operação de convolução linear é substituída pela convolução cíclica ou circular.

Neste trabalho, $x[n]$ denota uma seqüência com $N$ elementos pertencentes ao corpo dos reais. Existem operações básicas envolvendo tais seqüências, tais como a convolução cíclica, a transformada discreta de Fourier e a codificação em subbandas utilizando banco de filtros cíclicos.

Se $x[n]$ é a entrada de um sistema cíclico linear e invariante no tempo (CLIT) com resposta ao impulso $h[n]$, então sua saída é dada por [6]

$$
y[n]=\sum_{r=0}^{N-1} x[r] h\left[((n-r))_{N}\right],
$$

onde $((m))_{N}$ denota $m(\bmod N)$. A seqüência $y[n]$ é denominada a convolução cíclica ou circular de comprimento $N$ entre $x[n]$ e $h[n]$.

G. J. da Silva Jr. e R. M. Campello de Souza, Grupo de Processamento de Sinais, Departamento de Eletrônica e Sistemas, Universidade Federal de Pernambuco, Recife, PE, E-mails: gilsonjr@gmail.com, ricardo@ufpe.br.
As seqüências $x[n]$ e $X[k], n, k=0,1, \ldots, N-1$, onde $x[n] \in \mathbb{R}$ e $X[k] \in \mathbb{C}$, formam um par da transformada discreta de Fourier (TDF), denotado por

$$
x[n] \stackrel{\mathcal{F}}{\longleftrightarrow} X[k],
$$

se

$$
X[k] \triangleq \sum_{n=0}^{N-1} x[n] W_{N}^{k n},
$$

$$
x[n]=\frac{1}{N} \sum_{k=0}^{N-1} X[k] W_{N}^{-k n},
$$

onde $W_{N}=e^{-j 2 \pi / N}$. As propriedades da convolução cíclica e da TDF podem ser encontradas em [9].

Sistemas cíclicos (também chamados de filtros cíclicos) podem ser organizados em bancos de filtros cíclicos (BFC). A figura 1 mostra um BFC de dois canais. Para que ocorra reconstrução perfeita nessa estrutura, o filtro produto cíclico [10], $P[k] \triangleq H_{0}[k] G_{0}[k]$, deve satisfazer à condição

$$
P[k]+P\left[((k-N / 2))_{N}\right]=2,
$$

onde $N$ é um número par. Os outros filtros obedecem às relações

$$
H_{1}[k]=-W_{N}^{k d} G_{0}\left[((k-N / 2))_{N}\right]
$$

e

$$
G_{1}[k]=-W_{N}^{-k d} H_{0}\left[((k-N / 2))_{N}\right],
$$

onde $d$ é um inteiro ímpar.

Estas operações são facilmente estendidas para outras dimensões. A convolução cíclica 2D, a TDF2D e a codificação em sub-bandas 2D utilizando banco de filtros cíclicos, podem ser revistas no contexto de processamento de imagem.

Nesse contexto, uma imagem, $x[m, n]$, é uma matriz $M \mathrm{x} N$, onde $M$ e $N$ são inteiros pares. A convolução cíclica bidimensional entre duas matrizes $M \mathrm{x} N, x[m, n]$ e $h[m, n]$, é definida por [11], [12]

$$
\begin{gathered}
y[m, n] \triangleq \\
\sum_{r=0}^{M-1} \sum_{s=0}^{N-1} x[r, s] h\left[((m-r))_{M},((n-s))_{N}\right] .
\end{gathered}
$$

As matrizes $M \times 1 \times x[m, n]$ e $X[k, l], m, k=0,1, \ldots, M-1$ e $n, l=0,1, \ldots, N-1$, onde $x[m, n] \in \mathbb{R}$ e $X[k, l] \in \mathbb{C}$, formam um par TDF2D, denotado por

$$
x[m, n] \stackrel{\mathcal{F} 2 D}{\longleftrightarrow} X[k, l],
$$


(a)
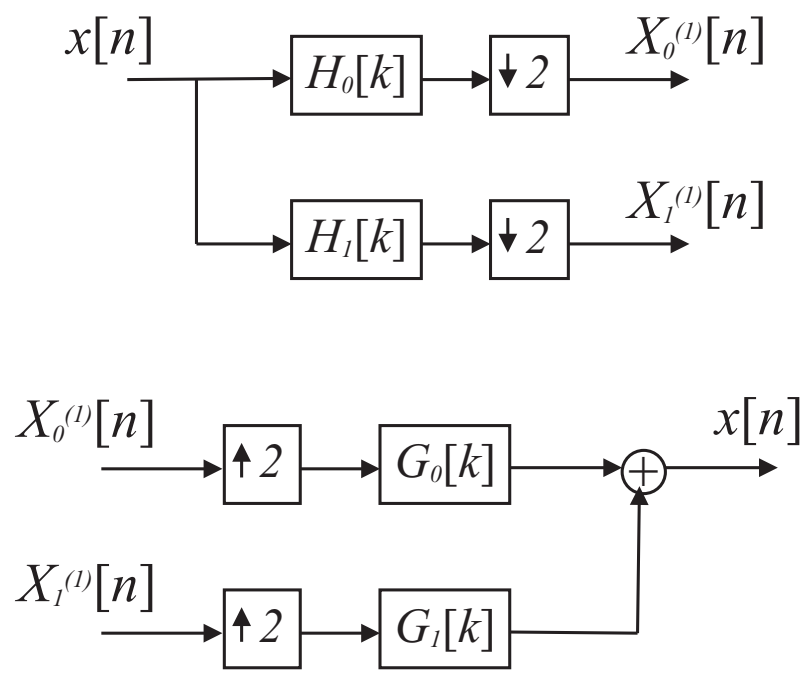

(b)

Fig. 1. Banco de filtros cíclicos com dois canais. (a) Banco de análise. (b) Banco de síntese.

se

$$
X[k, l] \triangleq \sum_{m=0}^{M-1} \sum_{n=0}^{N-1} x[m, n] W_{M}^{k m} W_{N}^{l n}
$$

e

$$
x[m, n]=\frac{1}{M N} \sum_{k=0}^{M-1} \sum_{l=0}^{N-1} X[k, l] W_{M}^{-k m} W_{N}^{-l n} .
$$

Aplicando a TDF2D em (7), o resultado é

$$
Y[k, l]=X[k, l] H[k, l],
$$

para $k=0,1, \ldots, M-1$ e $l=0,1, \ldots, N-1$, o que representa a multiplicação termo a termo das duas matrizes [12].

A codificação de imagem utilizando banco de filtros cíclicos 2D pode ser implementada utilizando-se um banco em todas as linhas e, em seguida, em todas as colunas. Nesse caso (o mais simples), os filtros 2D são ditos separáveis. A figura 2 mostra um banco de filtros cíclicos $2 \mathrm{D}$ com filtros separáveis. Os filtros de linha e coluna satisfazem à condição de reconstrução perfeita. A estrutura pode ser implementada utilizando-se decomposição polifásica cíclica [5], análoga à decomposição polifásica não cíclica [9], o que reduz significativamente a complexidade computacional. A análise multirresolução é feita como no caso não cíclico [1], [2].

Este trabalho esta organizado como segue. Na seção II são introduzidos os conceitos de filtros cíclicos e wavelets cíclicas de Shannon (WCS). Na seção III, estruturas de análise multirresolução são implementadas utilizando-se essas wavelets. Os resultados da interpolação de imagens utilizando a WCS são comparados com a interpolação utilizando a transformada discreta do cosseno tipo II (TDCII). Na seção IV é introduzido o conceito de filtro chave, para aplicação em segurança de dados. Uma aplicação para marca d'água e (a)
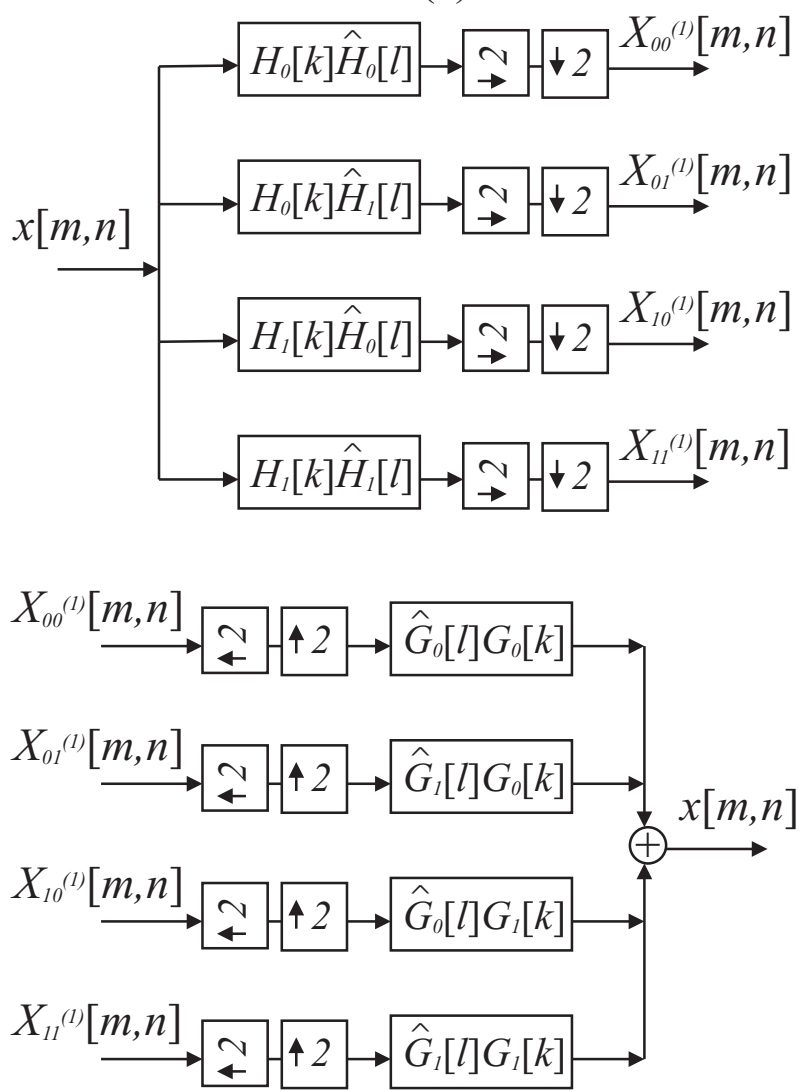

(b)

Fig. 2. Banco de filtros cíclicos 2D com filtros bidimensionais separáveis. Os sistemas subamostradores horizontal e vertical significam compressão em todas as colunas e em todas as linhas, respectivamente. Os sistemas sobreamostradores horizontal e vertical significam expansão em todas as colunas e em todas as linhas, respectivamente. (a) Banco de análise 2D. (b) Banco de síntese 2D.

esteganografia é apresentada. Na seção V estão as conclusões e observações finais.

\section{Filtros cíclicos de Shannon}

Definição 1: O filtro produto cíclico de Shannon, é definido por

$$
P_{s}[k] \triangleq\left\{\begin{array}{ll}
2, & 0 \leq k<N / 4 \text { e } 3 N / 4<k<N \\
1, & k=N / 4 \text { e } k=3 N / 4 \\
0, & N / 4<k<3 N / 4
\end{array},\right.
$$

com $k=0,1, \ldots, N-1$. Se 4 não divide $N$, a segunda linha em (11) deve ser excluída. O comprimento do filtro, $N$, é par.

O termo Shannon vem das wavelets contínuas de Shannon (a transformada de Fourier das wavelets de Shannon é análoga à forma desse filtro no domínio da TDF) [3].

Teorema 1: O filtro produto cíclico de Shannon, $P_{s}[k]$, satisfaz

$$
P_{s}[k]+P_{s}\left[((k-N / 2))_{N}\right]=2,
$$

para todo $N$ par, $k=0,1, \ldots, N-1$.

Demonstração: Deslocando-se ciclicamente $P_{s}[k]$ por $N / 2$, os valores 2 e 0 trocam de posição, enquanto que os 
valores 1 permanecem em suas posições originais. Somandose a seqüência deslocada com a seqüência original, o resultado segue.

Teorema 2: A TDF inversa do filtro produto cíclico de Shannon, $P_{s}[k]$, é dada por

$$
p_{s}[n]=\frac{2}{N} \frac{\operatorname{sen}(\pi n(N / 2+1) / N)}{\operatorname{sen}(\pi n / N)}-\frac{2}{N} \cos (\pi n / 2) .
$$

Demonstração: Basta calcular a TDF inversa a partir da definição.

Definição 2: Um banco de filtros cíclicos de Shannon (BFCS) tem os filtros de análise e síntese definidos pelas seguintes equações:

$$
\begin{gathered}
H_{0}[k]=\sqrt{P_{s}[k] / 2} \\
G_{0}[k]=2 H_{0}[k], \\
H_{1}[k]=-W_{N}^{k d} G_{0}\left[((k-N / 2))_{N}\right]
\end{gathered}
$$

$\mathrm{e}$

$$
G_{1}[k]=-W_{N}^{-k d} H_{0}\left[((k-N / 2))_{N}\right],
$$

onde $P_{s}[k]$ é o filtro produto cíclico de Shannon e $d$ é um inteiro ímpar.

Esses filtros foram usados em um projeto de BFC, com filtros simétricos de meia amostra, aplicados à compressão de imagem [13]. Pode-se dizer com isso que as wavelets cíclicas de Shannon possuem esse tipo de simetria e potencial de aplicações em compressão de imagem.

O BFCS depende apenas do filtro $P_{s}[k]$. Assim, o filtro passa-baixa de análise $H_{0}[k]$ é dado por

$$
H_{0}[k]= \begin{cases}1, & 0 \leq k<N / 4 \text { e } 3 N / 4<k<N \\ 1 / \sqrt{2}, & k=N / 4 \text { e } k=3 N / 4 \\ 0, & N / 4<k<3 N / 4\end{cases}
$$

No domínio do tempo, as equações ficam

$$
\begin{array}{r}
h_{0}[n]=\frac{1}{N} \frac{\operatorname{sen}(\pi n(N / 2+1) / N)}{\operatorname{sen}(\pi n / N)} \\
-\frac{2-\sqrt{2}}{2 N} \cos (\pi n / 2), \\
g_{0}[n]=2 h_{0}[n], \\
h_{1}[n]=(-1)^{n} g_{0}\left[((n-d))_{N}\right]
\end{array}
$$

e

$$
g_{1}[n]=(-1)^{n} h_{0}\left[((n+d))_{N}\right],
$$

onde o valor $d$ pode ser qualquer inteiro ímpar. Geralmente escolhe-se $d=1$.

\section{A. Wavelets Cíclicas de Shannon}

A análise da transformada wavelet cíclica (TWC) é dada por

$$
X_{0}^{(J)}[l]=\sum_{n=0}^{N-1} x[n] h_{0}^{(J)}\left[\left(\left(2^{J} l-n\right)\right)_{N}\right]
$$

$\mathrm{e}$

$$
X_{1}^{(j)}[l]=\sum_{n=0}^{N-1} x[n] h_{1}^{(j)}\left[\left(\left(2^{j} l-n\right)\right)_{N}\right],
$$

onde $j=1,2, \ldots, J, l=0,1, \ldots,\left(N / 2^{j}\right)-1$ e $J$ é o máximo valor de $j$ tal que $2^{j}$ divide $N$. A equação de síntese da TWC é

$$
\begin{aligned}
x[n]= & \sum_{j=1}^{J} \sum_{l=0}^{N / 2^{j}-1} X_{1}^{(j)}[l] g_{1}^{(j)}\left[\left(\left(n-2^{j} l\right)\right)_{N}\right] \\
& +\sum_{l=0}^{N / 2^{J}-1} X_{0}^{(J)}[l] g_{0}^{(J)}\left[\left(\left(n-2^{J} l\right)\right)_{N}\right] ;
\end{aligned}
$$

as seqüências $h_{0}^{(J)}[n]$ e $h_{1}^{(j)}[n]$ são chamadas de wavelets cíclicas de análise e as seqüências $g_{0}^{(J)}[n]$ e $g_{1}^{(j)}[n]$ são as wavelets cíclicas de síntese. Utilizando um BFCS para definir as wavelets cíclicas de Shannon (WCS), o resultado é

$$
\begin{gathered}
H_{0}^{(J)}[k]=\prod_{i=0}^{J-1} H_{0}\left[\left(\left(2^{i} k\right)\right)_{N}\right], \\
H_{1}^{(j)}[k]=H_{1}\left[\left(\left(2^{j-1} k\right)\right)_{N}\right] \prod_{i=0}^{j-2} H_{0}\left[\left(\left(2^{i} k\right)\right)_{N}\right], \\
G_{0}^{(J)}[k]=\prod_{i=0}^{J-1} G_{0}\left[\left(\left(2^{i} k\right)\right)_{N}\right]
\end{gathered}
$$

$$
G_{1}^{(j)}[k]=G_{1}\left[\left(\left(2^{j-1} k\right)\right)_{N}\right] \prod_{i=0}^{j-2} G_{0}\left[\left(\left(2^{i} k\right)\right)_{N}\right]
$$

onde $H_{0}[k], H_{1}[k], G_{0}[k]$ e $G_{1}[k]$ são filtros cíclicos de um BFCS e as WCS são obtidas pela TDF inversa

$$
h_{i}^{(j)}[n] \stackrel{\mathcal{F}}{\longleftrightarrow} H_{i}^{(j)}[k],
$$

e

$$
g_{i}^{(j)}[n] \stackrel{\mathcal{F}}{\longleftrightarrow} G_{i}^{(j)}[k] .
$$

A equação de síntese da TWC é utilizada para análise multirresolução e compactação de dados.

\section{ANÁlise MUltirResoluÇão COM WAVELETS CÍCLICAS DE SHANNON}

O conceito de análise multirresolução tem cinco axiomas e pode ser adaptado para sistemas de tempo discreto [1]. Esse conceito é aqui utilizado para análise multirresolução de seqüências cíclicas.

Definição 3: A componente de resolução $r$ da seqüência $x[n]$, de comprimento $N$, é definida por

$$
\begin{aligned}
x^{(r)}[n] \triangleq & \sum_{l=0}^{N / 2^{J}-1} X_{0}^{(J)}[l] g_{0}^{(J)}\left[\left(\left(n-2^{J} l\right)\right)_{N}\right] \\
& +\sum_{j=r}^{J} \sum_{l=0}^{N / 2^{j}-1} X_{1}^{(j)}[l] g_{1}^{(j)}\left[\left(\left(n-2^{j} l\right)\right)_{N}\right],
\end{aligned}
$$

onde $r=1,2, \ldots, J+1$ e $g_{i}^{(j)}[n]$ são wavelets cíclicas de análise. 
Nesse caso, existem $J+1$ resoluções diferentes, onde a resolução máxima é $r=1$. Com essa definição,

$$
x^{(J+1)}[n]=\sum_{l=0}^{N / 2^{J}-1} X_{0}^{(J)}[l] g_{0}^{(J)}\left[\left(\left(n-2^{J} l\right)\right)_{N}\right],
$$

e

$$
x^{(1)}[n]=x[n] .
$$

Sendo $V_{j}$ o espaço vetorial gerado por $g_{0}^{(j)}\left[\left(\left(n-2^{j} l\right)\right)_{N}\right] \mathrm{e}$ $W_{j}$ o espaço vetorial gerado por $g_{1}^{(j)}\left[\left(\left(n-2^{j} l\right)\right)_{N}\right]$, pode-se escrever que

$$
V_{J+1} \subset V_{J} \subset \ldots V_{2} \subset V_{1}
$$

$\mathrm{e}$

$$
V_{j-1}=V_{j} \oplus W_{j}
$$

A componente $x^{(2)}[n]$ pode ser vista como uma interpolação de $X_{0}^{(1)}[n]$, a qual é uma seqüiência com comprimento $N / 2$, e a componente $x^{(3)}[n]$ pode ser vista como uma interpolação de $X_{0}^{(2)}[n]$, que tem comprimento $N / 4$.

Para a análise de imagens com a WCS, as operações ocorrem em todas as linhas e todas as colunas da imagem. Desse modo, $x^{(2)}[m, n]$ é uma interpolação de uma imagem com área quatro vezes menor (metade das linhas e metade das colunas) e assim por diante.

$\mathrm{Na}$ Figura 3 é mostrado um exemplo de análise multirresolução com a WCS.

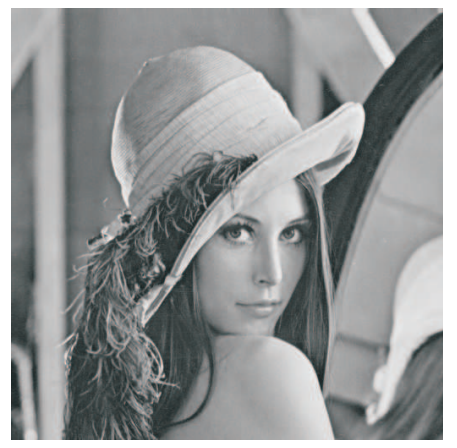

(a)

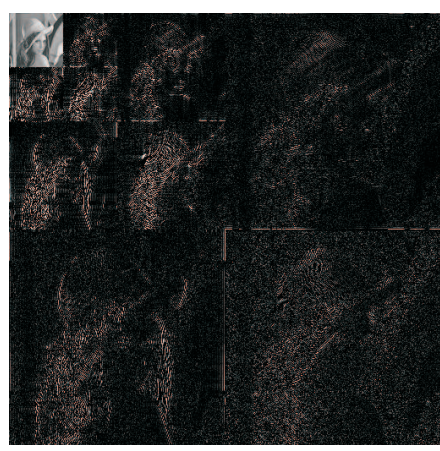

(b)
Fig. 3. Análise multirresolução. (a) Imagem original. (b) Análise multirresolução com 3 estágios (os níveis de detalhe tiveram um ganho de 10 para visualização).

\section{A. Interpolação $W C S 2 D$ versus $D C T 2 D$}

A transformada discreta do cosseno tipo II (DCTII) é uma das transformadas mais utilizadas para compactação de energia e interpolação [9], [12], sendo usada em padrões de compactação de imagem e vídeo como o JPEG e o MPEG [14].

A WCS2D foi comparada com a DCT2D tipo II, os resultados estão mostrados na Tabela I. O símbolo * indica que a análise foi feita na parte central da imagem, equivalente a $90 \%$ da imagem total. Isso foi feito para atenuar (ou desconsiderar)
TABELA

RESULTADOS DA INTERPOLAÇÃO.

\begin{tabular}{|l|c|c|c|c|}
\hline Imagem & MSE dct & MSE wcs & Emax dct & Emax wcs \\
\hline Lena i4 & 16.2453 & 18.8749 & 48.7769 & 48.5702 \\
Lena i4* & 17.2212 & 17.1955 & 48.7769 & 48.5702 \\
Lena i16 & 66.6901 & 72.7921 & 101.8129 & 100.7620 \\
Lena i16* & 71.8659 & 71.6277 & 101.8129 & 100.7620 \\
Elaine i4 & 28.4176 & 32.0646 & 48.0099 & 94.7942 \\
Elaine i4* & 29.3935 & 29.4451 & 48.0099 & 47.8994 \\
Elaine i16 & 48.5390 & 57.1365 & 67.2422 & 120.9367 \\
Elaine i16* & 50.6354 & 50.7651 & 67.2422 & 68.0782 \\
\hline
\end{tabular}

o problema da distorção nas bordas. O código "i $\alpha$ " significa interpolação da imagem com área $\alpha$ vezes menor.

Na Figura 4 está mostrada uma interpolação "i16" com a WCS. Fica evidente a distorção nas bordas da imagem. Entretanto, 90\% da área central da imagem fornece resultados comparáveis aos da DCT (Em alguns casos o resultado é melhor). As figuras de mérito utilizadas são o MSE (erro médio quadrático) e o $E_{\max }$ (erro máximo) que são dados respectivamente por

$$
\operatorname{MSE}=\frac{1}{M N} \sum_{m=0}^{M-1} \sum_{n=0}^{N-1}\left(x[m, n]-x^{\prime}[m, n]\right)^{2}
$$

e

$$
E_{\max }=\max _{(0 \leq m<M)(0 \leq n<N)}\left|x[m, n]-x^{\prime}[m, n]\right| .
$$

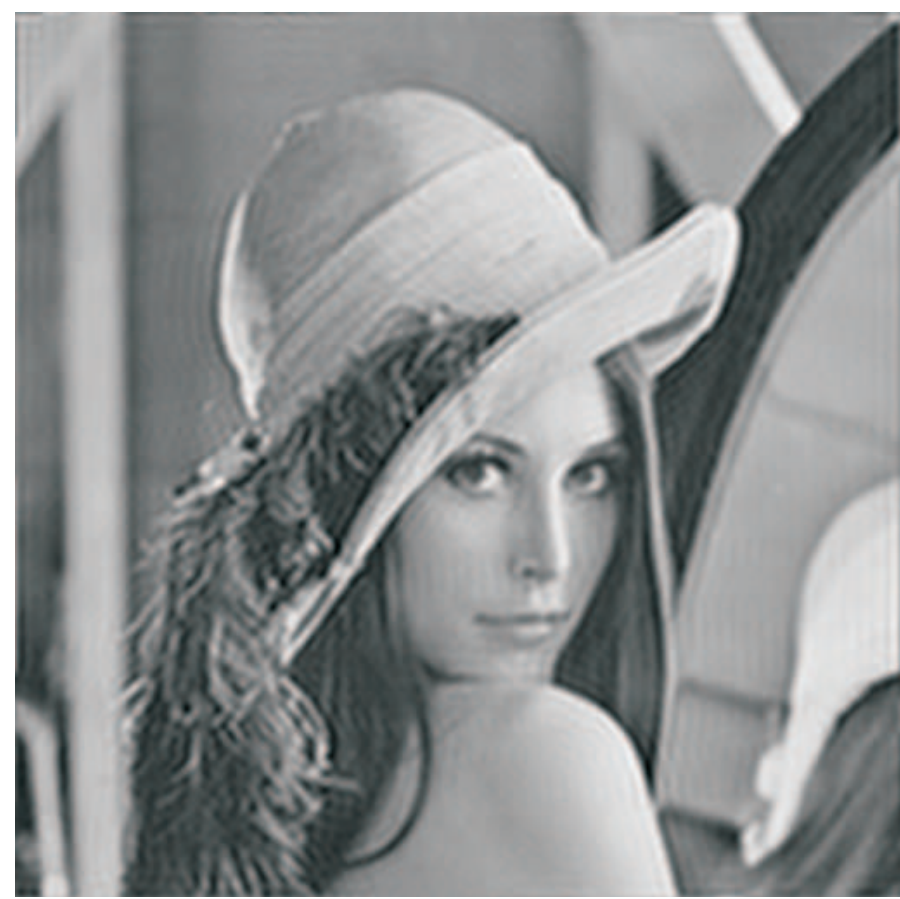

Fig. 4. Interpolação "i16" da imagem Lena utilizando a WCS.

A conclusão é que a capacidade de interpolação e compactação da WCS é comparável à da DCTII. Como a complexidade computacional da WCS é menor que a da DCTII, isso a torna uma ferramenta atrativa para sistemas de compactação que requerem maior velocidade. Além disso, as wavelets cíclicas de Shannon podem ser usadas em 
substituição às wavelets não cíclicas empregadas em sistemas de compactação atuais [2], [14].

\section{O Filtro Chave}

No que se segue é introduzido uma nova classe de filtros com recuperação perfeita para utilização em codificação de imagem, marca d'água e esteganografia.

Definição 4: Um filtro produto chave é um filtro cíclico, de comprimento $N$ par, da forma

$$
P_{c}[k] \triangleq\left\{\begin{array}{ll}
2 b[0], & k=0 \\
2(1-b[0]), & k=N / 2 \\
1, & k=N / 4 ; \quad k=3 N / 4 \\
2 b[i], & k=i ; \quad k=N-i \\
2(1-b[i]), & k=i+N / 2 ; \quad k=N / 2-i
\end{array},\right.
$$

onde $1 \leq i<N / 4$, e a chave, $b[i], i=0,1, \ldots, N / 4-1$, é um vetor binário qualquer. Se 4 não divide $N$, desconsidera-se a terceira linha da definição.

Observa-se que $P_{s}[k]$ é um caso particular de filtro produto chave quando $b[i]$ é a sequiência toda 1 . O filtro chave é completamente especificado pela chave $b[i]$.

Teorema 3: Todo filtro produto chave, $P_{c}[k]$, satisfaz

$$
P_{c}[k]+P_{c}\left[((k-N / 2))_{N}\right]=2 .
$$

Demonstração: Fazendo-se o deslocamento cíclico de $N / 2$, a primeira linha da definição permuta com a segunda, a terceira permanece e a quarta permuta com a quinta. Somandose com a seqüência original, o resultado segue.

Definição 5: Os filtros chave de análise, $h_{0}[n]$ e $h_{1}[n]$, e os filtros chave de síntese, $g_{0}[n]$ e $g_{1}[n]$, são definidos a partir de um filtro produto chave $P_{c}[k]$ e são dados por

$$
\begin{gathered}
h_{0}[n] \triangleq \frac{1}{N} \sum_{k=0}^{N-1} \sqrt{P_{c}[k] / 2} W_{N}^{-n k}, \\
g_{0}[n] \triangleq 2 h_{0}[n], \\
h_{1}[n] \triangleq(-1)^{n} g_{0}\left[((n-d))_{N}\right]
\end{gathered}
$$

$\mathrm{e}$

$$
g_{1}[n] \triangleq(-1)^{n} h_{0}\left[((n+d))_{N}\right],
$$

onde o valor $d$ pode ser qualquer inteiro ímpar.

Teorema 4: Os filtros chave de análise e síntese são reais.

Demonstração: Vem da propriedade da TDF, pois $H_{0}[k]=\sqrt{P_{c}[k] / 2}$ é real e par, portanto, sua transformada inversa, $h_{0}[n]$, é real e par. Todos os outros filtros dependem de $h_{0}[n]$ através de funções reais, o que completa a prova.

Está claro que filtros chaves de análise e síntese formam um banco de filtros cíclicos com reconstrução perfeita. A análise com esse banco é totalmente dependente da chave, $b[i]$, e os resultados são bem "aleatórios".

\section{A. Aplicações em esteganografia e marca d'água}

Para a análise de imagem com esses bancos, tem-se as opções: i) Utilizar uma chave para todas as linhas e outra para todas as colunas (nessa situação, o comprimento da chave é $(M+N) / 4$ bits); ii) Utilizar chaves diferentes para cada linha e para cada coluna (o comprimento da chave é $(M N / 2)$ bits; a complexidade aumenta pois os filtros bidimensionais tornam-se não separáveis), o que pode aumentar a segurança em relação à primeira opção.

Para simples demonstração, será considerada a mesma chave para todas as linhas e todas as colunas (utilizando uma imagem quadrada). Primeiro faz-se uma análise multirresolução com os filtros chave de análise. Na Figura 5 é mostrada a imagem de Elaine com sua análise de três estágios, utilizando-se o banco de análise com uma chave arbitrária. Em seguida, é adicionada

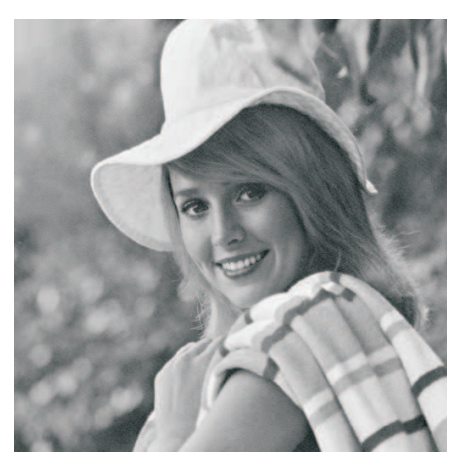

(a)

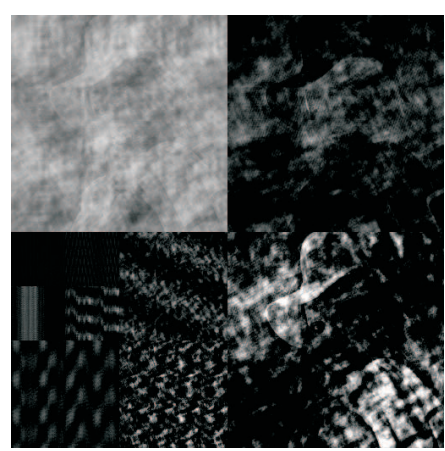

(b)
Fig. 5. Análise da imagem utilizando filtros chave de análise. (a) Imagem original. (b) Resultado da análise com os filtros chave.

uma marca d'água ou mensagem secreta (dependendo da aplicação) em uma das sub-bandas (substituindo a posição original), como mostra a Figura 6. Finalmente, utiliza-se o filtro

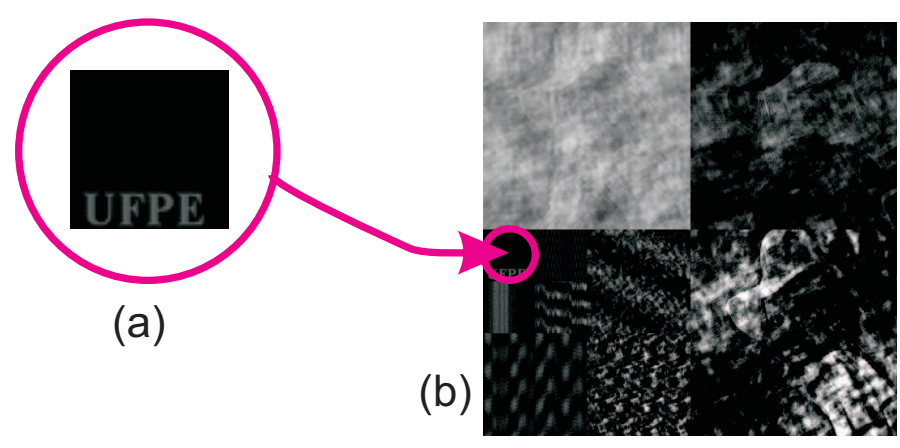

Fig. 6. Inserção da informação (marca d'água ou estego mensagem). (a) Imagem a ser adicionada em uma das sub-bandas da imagem original. (b) Sub-banda alterada com a informação inserida.

chave de síntese para gerar uma imagem contendo informação. A Figura 7 mostra o resultado final. Para a imagem inserida ser recuperada é necessário o conhecimento da chave $b[i]$. Também é possível uma grande variedade de combinações para aumentar a segurança; como exemplo, pode-se sugerir a inserção de imagens falsas, inseridas com diferentes chaves na imagem original; utilizar corpos finitos e inserir funções 


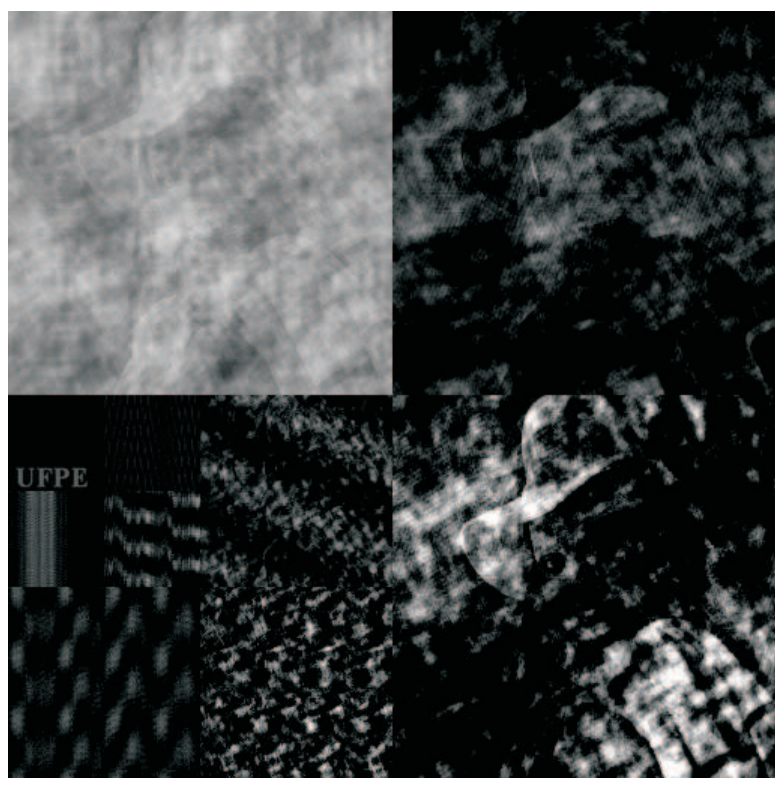

(a)

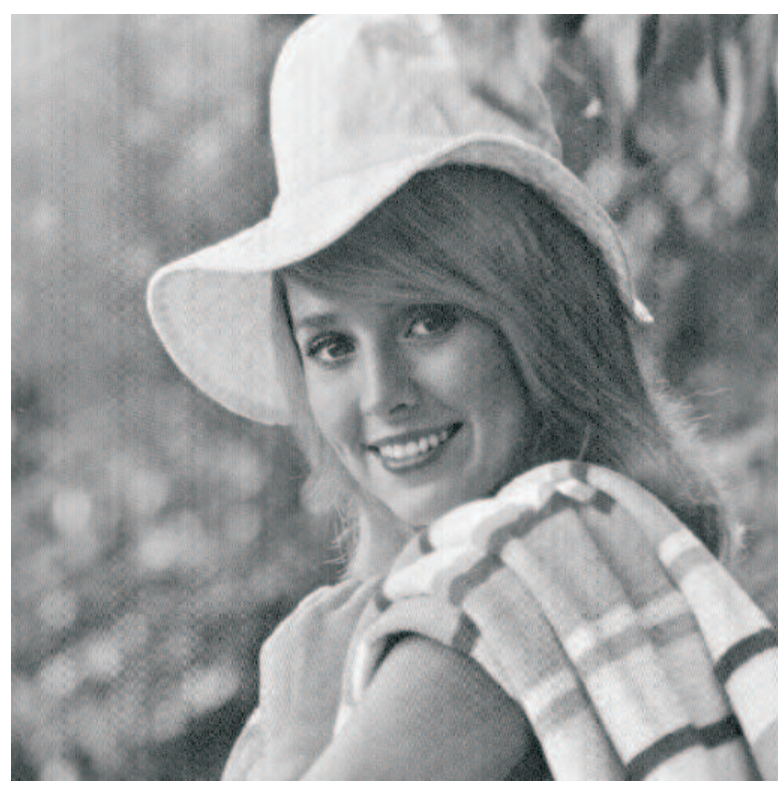

(b)

Fig. 7. Resultado da síntese com os filtros chave. (a) Entradas para o banco de síntese com os filtros chave. (b) Resultado da reconstrução da imagem com a informação inserida.

não lineares para aumentar a segurança, como em [15]. A segurança desse exemplo ainda não foi analisada, o esquema é apenas uma sugestão inicial para a implementação de sistemas mais robustos.

\section{Conclusões}

Nesse artigo os bancos de filtros cíclicos foram revistos e as wavelets cíclicas de Shannon (WCS) foram introduzidas. Aplicações em processamento de imagem foram apresentadas para essas ferramentas. A interpolação utilizando-se a WCS foi comparada com a interpolação realizada por meio da transformada discreta do cosseno tipo II e obteve resultados satisfatórios, os quais indicam que a capacidade de interpolação e compactação da WCS é comparável à da DCTII. O conceito de filtro chave foi introduzido, com aplicações em segurança de dados. Um exemplo de inserção de marca d'água foi apresentado utilizando filtros chave. Métodos de compactação de imagem baseados na WCS estão sendo investigados. Além disso, outras wavelets cíclicas estão sendo investigadas para aplicações em multiplexação de dados, processamento de imagem e processamento de áudio.

\section{AgRADECIMENTOS}

Os autores agradecem ao Prof. Dr. Hélio M. de Oliveira por suas valorosas sugestões ao presente trabalho. O primeiro autor agradece ao $\mathrm{CNPq}$.

\section{REFERÊNCIAS}

[1] M. Vetterli and J. Kovacevic, Wavelets and Subband Coding. Prentice Hall, 1995.

[2] G. Strang and T. Nguyen, Wavelets and Filter Banks. Wellesley Cambridge Press, 1997.
[3] H. M. de Oliveira, Análise de Sinais para Engenheiros: uma abordagem via Wavelets. Brasport, 2007.

[4] P. P. Vaidyanathan and A. Kirac, "Theory of cyclic filter banks," in ICASSP '97: Proceedings of the 1997 IEEE International Conference on Acoustics, Speech, and Signal Processing (ICASSP '97)-Volume 3. Washington, DC, USA: IEEE Computer Society, 1997, p. 2449.

[5] _ "Cyclic LTI systems and the paraunitary interpolation problem," IEEE Transactions on Signal Processing, vol. 3, pp. 1445-1448, May 1998.

[6] _ "Cyclic LTI systems in digital signal processing," IEEE Transactions on Signal Processing, vol. 47, pp. 433-447, Feb. 1999.

[7] G. Caire, R. L. Grossman, and H. V. Poor, "Wavelet transforms associated with finite cyclic groups," IEEE Transactions on Information Theory, vol. 39, no. 4, pp. 1157-1166, July 1993.

[8] H. Murakami, "Discrete wavelet transform based on cyclic convolutions," IEEE Transactions on Signal Processing, vol. 52, pp. 165- 174, Jan. 2004.

[9] A. V. Oppenheim, R. W. Schafer, and J. R. Buck, Discrete-Time Signal Processing, 2nd ed. Prentice Hall, 1999.

[10] G. J. da Silva Jr. and R. M. C. de Souza, "Banco de filtros e wavelets para sistemas cíclicos sobre corpos finitos," XXV Simpósio Brasileiro de Telecomunicações - SBrT 2007, Setembro 2007, em CD.

[11] R. E. Blahut, Fast Algorithms for Digital Signal Processing. AddisonWesley Publishing Company, 1984.

[12] J. S. Lim, Two-Dimensional Signal and Image Processing. Prentice Hall, 1990.

[13] K. Ichiwara, T. Uto, T. Morimoto, and O. Kenji, "On two-channel orthogonal cyclic filter banks with half-sample symmetric filters," 6th International Conference on Information, Communications and Signal Processing, 2007, pp. 1-4, Dec. 2007.

[14] V. Bhaskaran and K. Konstantinides, Image and Video Compression Standards: Algorithms and Architectures, 2nd ed. Kluwer Academic Publishers, 1997.

[15] K. S. Chan and F. Fekri, "A block cipher cryptosystem using wavelet transforms over finite fields," IEEE Transactions on Signal Processing, vol. 52, no. 10, pp. 2975-2991, October 2004. 